\title{
AN INCONSISTENCY OF THE METHOD OF MAXIMUM LIKELIHOOD
}

\author{
By D. BASU \\ University of California, Berkeley
}

An example was given by Neyman and Scott [2] to show that there are situations where the method of maximum likelihood leads to inconsistent estimators. In their example considered, the observations were supposed to be drawn from an infinite sequence of distinct populations involving an infinite sequence of nuisance parameters.

An example is given here to demonstrate that even in simple situations where all the observations are independently and identically distributed and involve only one unknown parameter, the method of maximum likelihood may lead us astray. The example typifies the situations where the correct method of setting up a point estimate should begin with a test of hypothesis between two composite alternatives.

Let $A$ be the set of all rational numbers in the closed interval $(0,1)$ and $B$ any countable set of irrational numbers in the same interval. Let $X$ be a random variable that takes the two values 0 and 1 with

$$
P(X=1)= \begin{cases}\theta & \text { if } \theta \varepsilon A, \\ 1-\theta & \text { if } \theta \varepsilon B .\end{cases}
$$

If $r$ is the total number of 1 's in a set of $n$ random observations on $X$, then from the rationality of $r / n$ it follows at once that the maximum likelihood estimator of $\theta$ is $r / n$. But $r / n$ converges (in probability) to $\theta$ or $1-\theta$ according as $\theta \varepsilon A$ or $\theta \varepsilon B$.

Now, since $A$ and $B$ are both countable sets, it follows [1] that there exists a consistent test for the composite hypothesis $\theta \varepsilon A$ against the composite alterna-

Received March 19, 1954. 
tive $\theta \varepsilon B$. In other words, there exists a function $\varphi_{n}$ of the first $n$ observations such that $\varphi_{n}$ takes only the two values 0 and 1 and such that

$$
P\left(\varphi_{n}=1 \mid \theta\right) \rightarrow\left\{\begin{array}{ll}
0 & \text { if } \theta \varepsilon A, \\
1 & \text { if } \theta \varepsilon B,
\end{array} \quad n \rightarrow \infty .\right.
$$

It then follows readily that the estimator

$$
t_{n}=\left(1-\varphi_{n}\right) r / n+\varphi_{n}(1-r / n)
$$

is a consistent estimator of $\theta$. Thus, though there exist consistent estimators for $\theta$, the maximum likelihood estimator is not consistent.

For a simple construction for the function $\varphi_{n}$, let $\left\{\alpha_{i}\right\}$ and $\left\{\beta_{i}\right\}$, for $i=$ $1,2, \cdots$, be two enumerations of the sets $A$ and $B$, respectively, and let $\delta_{k}$ be the distance between the two sets $\left(\alpha_{1}, \cdots, \alpha_{k}\right)$ and $\left(1-\beta_{1}, \cdots, 1-\beta_{k}\right)$. Let $k(n)$ be the largest integer $k$ such that $\delta_{k}>n^{-1 / 4}$. Note that $k(n)$ increases monotonically to infinity. Let $I_{k n}$ and $J_{k n}$ be the open intervals of length $n^{-1 / 4}$ centered around $\alpha_{k}$ and $1-\beta_{k}$ respectively and let

$$
R_{n}=\bigcup_{k \leqq k(n)} I_{k n}, \quad S_{n}=\bigcup_{k \leqq k(n)} J_{k n} .
$$

For every $n$, the sets $R_{n}$ and $S_{n}$ are clearly disjoint. Now consider a fixed $k$. For all $n$ for which $k(n) \geqq k$ we have

$$
\begin{aligned}
P\left(r / n \varepsilon S_{n} \mid \theta=\beta_{k}\right) & \geqq P\left(r / n \varepsilon J_{k n} \mid \theta=\beta_{k}\right) \\
& =P\left(\left|r / n-\left[1-\beta_{k}\right]\right|<-n^{-1 / 4} \mid \theta=\beta_{k}\right) \\
& \rightarrow 1 \text { as } n \rightarrow \infty,
\end{aligned}
$$

because $r / n$ is asymptotically normal with mean $1-\beta_{k}$ and asymptotic s.d. $\sqrt{\beta_{k}\left(1-\beta_{k}\right) / n}$. By the same argument we have

$$
\begin{aligned}
P\left(r / n \varepsilon S_{n} \mid \theta=\alpha_{k}\right) & \leqq 1-P\left(r / n \varepsilon R_{n} \mid \theta=\alpha_{k}\right) \\
& \rightarrow 0 \text { as } n \rightarrow \infty .
\end{aligned}
$$

Now, if we define

$$
\varphi_{n}= \begin{cases}1 & \text { if } r / n \varepsilon S_{n} \\ 0 & \text { otherwise, }\end{cases}
$$

then $\varphi_{n}$ clearly has the required property.

Acknowledgement. I wish to thank Mr. J. L. Hodges, Jr., for his help in the writing of this note.

\section{REFERENCES}

[1] Charles Kraft, "On the problem of consistent and uniformly consistent statistical procedures", Unpublished Ph.D. thesis submitted to the University of California (1954).

[2] J. Nexman and E. L. Scotr, "Consistent estimates based on partially consistent observations," Econometrica, Vol. 16 (1948), pp. 1-32. 